7 Bärtsch $\mathrm{P}$, Mairbäurl $\mathrm{H}$, Maggiorini $\mathrm{M}$, et al. Physiological aspects of high-altitude pulmonary edema. J Appl Physiol 2005; 98: 1101-1110.

8 Dehnert C, Risse F, Ley S, et al. Magnetic resonance imaging of uneven pulmonary perfusion in hypoxia in humans. Am J Respir Crit Care Med 2006; 174: 1132-1138.

9 Naeije R, De Backer D, Vachiéry JL, et al. High-altitude pulmonary edema with primary pulmonary hypertension. Chest 1996; 110: 286-289.
10 Galié N, Hoeper MM, Humbert M, et al. Guidelines for the diagnosis and treatment of pulmonary hypertension. The Task Force for the Diagnosis and Treatment of Pulmonary Hypertension of the European Society of Cardiology (ESC) and the European Respiratory Society (ERS), endorsed by the International Society of Heart and Lung Transplantation (ISHLT). Eur Respir J 2009; 34: 1219-1263.

DOI: $10.1183 / 09031936.00200409$

\title{
Pulmonary alveolar proteinosis associated with Epstein-Barr virus infection
}

To the Editors:

We report the case of a 3-yr-old male who became unwell over a period of months. He was found to have pulmonary alveolar proteinosis (PAP), confirmed by computed tomography (CT) scan, bronchoalveolar lavage fluid and lung biopsy. Histology from the lung biopsy also revealed a high load of Epstein-Barr virus (EBV). EBV DNA was also found in the blood samples by PCR. We believe this is the first case of PAP caused by EBV reported in the literature.

A 3-yr-old male, born at 34 weeks gestation, had transient infantile hypothyroidism. He had been discharged from an endocrinology clinic at 2 yrs of age, but re-presented with failure to thrive. He was the second twin of consanguineous Asian parents, who had lived in the UK for 15 yrs. They had three healthy older daughters. On examination, he was tachypnoeic and tachycardic with signs of respiratory distress. He had persistent hypoxaemia despite high-flow oxygen and was admitted to the Paediatric Intensive Care Unit (Sheffield Children's Hospital, Sheffield, UK). Chest radiography showed bilateral air-space opacification, and he was administered i.v. cefuroxime. Within $12 \mathrm{~h}$, his condition deteriorated and endotracheal mechanical ventilation was commenced. An initial diagnosis of pneumonia, possibly due to an atypical organism or tuberculosis, was made and the antibiotic spectrum was widened to include clarithromycin, cotrimoxazole, clindamycin, rifampicin, isoniazid, pyridoxine, pyrazonamide and fluconazole.

A blind tracheobronchial lavage was performed by the intensive-care staff. The aspirated fluid was reported to be clear and a silver stain was negative for Pneumocystis jiroveci, but rhinovirus was detected by PCR; there was no significant bacterial growth. The lavage was negative for influenza A and $B$, parainfluenza 1, 2, and 3, respiratory syncytial virus, and adenovirus. A respiratory review 1 week after admission raised the question of interstitial lung disease, and a high-resolution CT scan of the chest and a lung biopsy were performed.

The CT scan (fig. 1) showed severe, bilateral, widespread interstitial opacities with generalised, interlobular septal thickening and ground-glass opacities, with bilateral dependent atelectasis. The changes were felt to be nonspecific, although suggestive of PAP.
PCR blood tests for EBV were positive, with "raised" EBV immunoglobulin (Ig)M and IgG (levels not reported). The histology of the lung biopsy (fig. 2) showed alveolar spaces containing Periodic acid-Schiff-positive eosinophilic proteinaceous granular material, foamy macrophages and cholesterol clefts. In the absence of inflammation and/or interstitial fibrosis, these features were consistent with PAP. Electron microscopy examination revealed that the proteinaceous material was composed of $0.7-\mu \mathrm{m}$, tightly packed myelinoid material. Further blood tests excluded known mutations in genes for surfactant proteins B and C, ABCA3 (adenosine triphosphate-binding cassette subfamily A member 3), SLAM (signalling lymphocyte-activation molecule)-associated protein and granulocyte-macrophage colony-stimulating factor (GM$\mathrm{CSF}$ ) receptor. T-cell clonality was normal and serology to human herpesvirus 6 and adenovirus were negative. Serum Ig levels, vaccine responses and lymphocyte subsets were normal, as was urine lysine.

EBV DNA was demonstrated in the lung tissue by PCR $\left(20,000\right.$ copies $\left.\cdot \mathrm{mL}^{-1}\right)$, and was also found in blood samples from day 3, 8 and 11 of the illness $\left(118,291\right.$ and 511 copies $\cdot \mathrm{mL}^{-1}$,

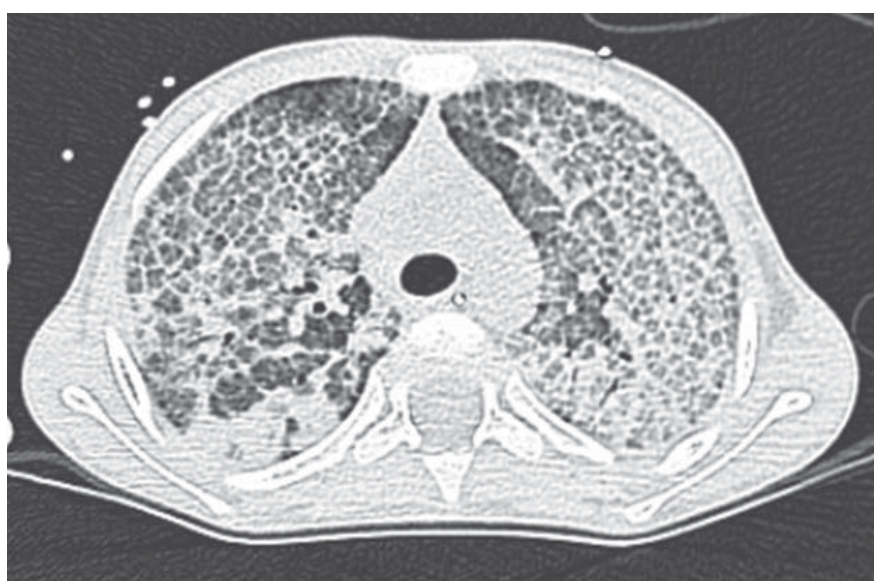

FIGURE 1. High-resolution computed tomography scan of chest, showing widespread interstitial opacities with generalised interlobular septal thickening and ground-glass opacities. 

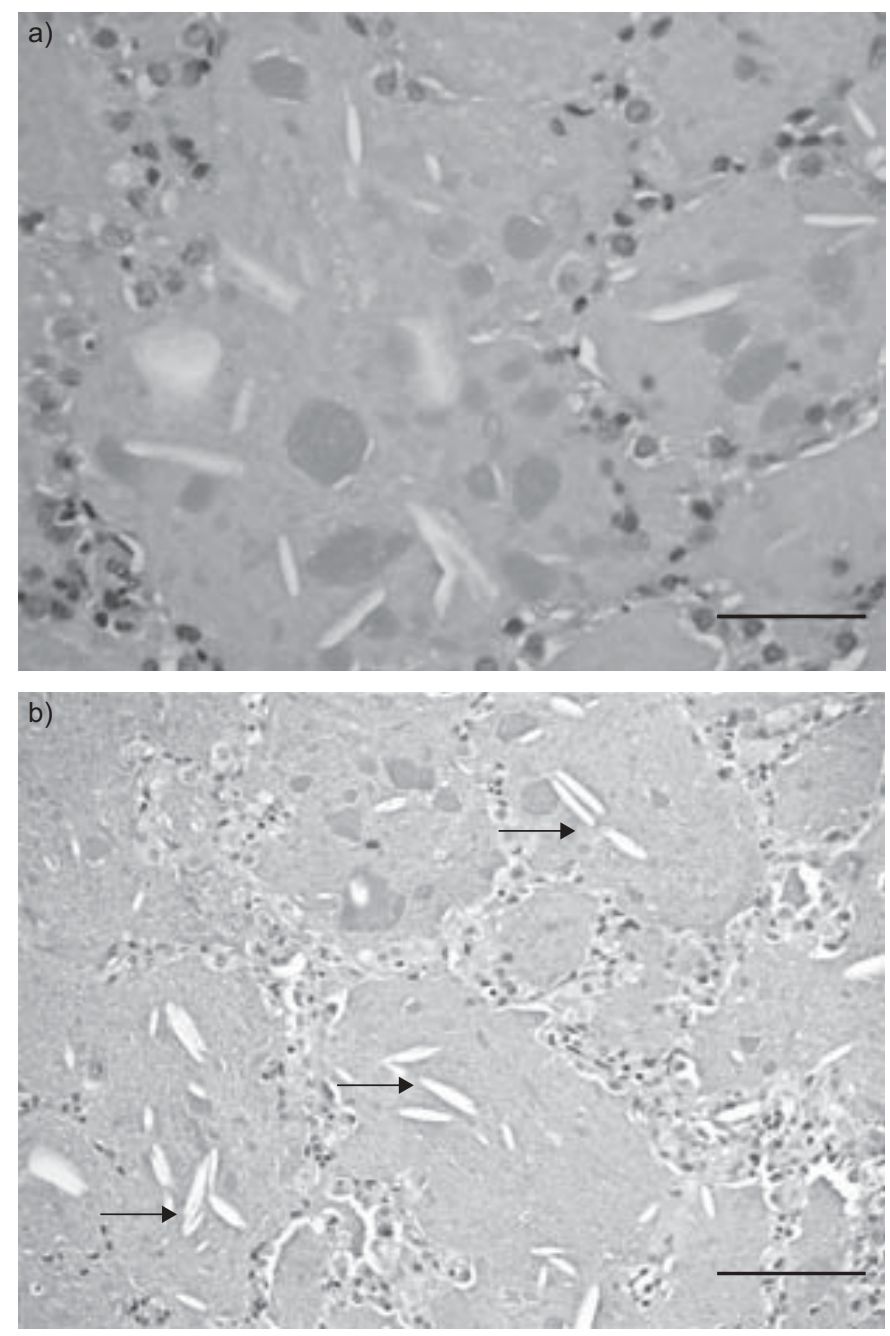

FIGURE 2. a) Microphotograph showing proteinaceous material diffusely filling alveolar spaces. Note the absence of inflammatory cell infiltrate. Periodic acidSchiff (PAS) stain. b) Cholesterol clefts (arrows) were seen at low magnification. PAS stain. Scale bar $=50 \mu \mathrm{m}$.

respectively). A diagnosis of PAP secondary to EBV was made. The antibiotics were rationalised to coamoxiclav, ciprofloxacin and fluconazole, and finally stopped. He was then administered ganciclovir. EBV was undetectable by PCR on subsequent blood samples on days 22, 29 and 43, and was detectable at $<250$ copies $\cdot \mathrm{mL}^{-1}$ in lung lavage on day 43 .

3 weeks after admission, his respiratory condition was unimproved and therapeutic lung lavage was initiated. Milky fluid was obtained from the lavage, clearing towards the end of each procedure. A total of five lung lavages were performed, initially segmental, and subsequently, unilateral whole lung lavages. These resulted in a slow but steady improvement in his condition, so that he was extubated after two lavages. During the final lavage, performed 70 days after the first one, the initial fluid return was much less milky than previous procedures. 1 week after the final procedure, he was breathing spontaneously in air and discharged from hospital. At discharge his weight had recovered from below the 0.4th centile to the 2 nd centile. He has remained well for 18 months. 12 months after his recovery he was tested for antibodies to GM-CSF, which were negative. Unfortunately, no sample from the acute illness was available for testing.

PAP was first described in 1958 [1]. PAP is a rare disorder characterised by the intra-alveolar accumulation of surfactant lipids and proteins impairing gas exchange and resulting in progressive respiratory insufficiency. It can be primary (also called acquired), secondary or congenital. Primary (acquired) PAP is largely due to an inhibitory antibody to GM-CSF [1]. Secondary PAP occurs as a consequence of any one of a heterogeneous group of underlying clinical conditions, which include: haematological malignancies; inhalation of toxic dust, fumes or gases; immunosuppression or immunodeficiency; and lysinuric protein intolerance. These conditions all result in alveolar surfactant accumulation, possibly by impairing alveolar macrophage function or responsiveness to GM-CSF [2]. Congenital PAP is due to mutations in the genes encoding surfactant proteins B or C, ABCA3 [3] or GM-CSF [4] receptor, and as the name suggests, presents in the neonatal period.

A clinical diagnosis of PAP can often be made by the characteristic milky colour of the bronchoalveolar lavage fluid. In our case, the initial tracheobronchial lavage was performed prior to respiratory referral, and described as a bronchoalveolar lavage with a clear return. A more accurate account of this procedure may have hastened the diagnosis.

Whole-lung lavage has been widely used as a treatment and has been associated with long-term survival [5]. Inhaled and subcutaneous GM-CSF have also been used with some success in acquired cases $[6,7]$ but is probably ineffective unless an autoantibody to GM-CSF is present. No immunodeficiency was found and no other known cause was found in the present case. The increase and decrease of EBV in the blood of our patient, and the high concentrations in lung tissue suggest that acute EBV was, at least temporally, associated with, and most likely to have been the cause of PAP in this case. Although infectious causes of secondary PAP have been identified, EBV has not been previously described as a cause, suggesting that this is the first reported case. The mechanism for transient PAP in conditions such as haematological malignancy is unclear. Since EBV infection is well known to stimulate a wide range of transient autoantibody responses [8], we suggest that the mechanism in our case may have been the transient induction of an anti-GM-CSF antibody, although this must remain no more than speculation, since no acute sample was retained for testing.

\section{Edwards*, R. Primhak* and M.C. Cohen ${ }^{\#}$}

*Dept of Respiratory Medicine, and \#Dept of Histopathology, Sheffield Children's NHS Foundation Trust, Sheffield, UK.

Correspondence: R. Primhak, Sheffield Children's Hospital, Western Bank, Sheffield, S10 2TH. E-mail: r.a.primhak@ sheffield.ac.uk

Statement of Interest: None declared. 
Acknowledgements: Thanks to S. Burdach and C. Goetz (both Laboratory for Functional Genomic and Transplantation Biology, Munich, Germany) for the exclusion of GM-CSF receptor mutations, and B. Trapnell and C. Stevens (Cincinnati Children's Hospital, Cincinnati, OH, USA) for measurement of anti-GM-CSF antibodies.

\section{REFERENCES}

1 Kitamura T, Uchida K, Tanaka N, et al. Serological diagnosis of idiopathic pulmonary alveolar proteinosis. Am J Respir Crit Care Med 2000; 162: 658-662.

2 Seymour JF, Presneill JJ. Pulmonary alveolar proteinosis: progress in the first 44 years. Am J Respir Crit Care Med 2002; 166: 215-235.

3 de Blic J. Pulmonary alveolar proteinosis in children. Paediatr Respir Rev 2004; 5: 316-322.
4 Dirksen U, Nishinakamura R, Groneck P, et al. Human pulmonary alveolar proteinosis associated with a defect in GM-CSF/IL-3/IL-5 receptor common $\beta$ chain expression. J Clin Invest 1997; 100: 2211-2217.

5 Beccaria M, Luisetti M, Rodi G, et al. Long-term durable benefit after whole lung lavage in pulmonary alveolar proteinosis. Eur Respir J 2004; 23: 526-531.

6 Seymour JF, Presneill JJ, Schoch OD, et al. Therapeutic efficacy of granulocyte-macrophage colony-stimulating factor in patients with idiopathic acquired alveolar proteinosis. Am J Respir Crit Care Med 2001; 163: 524-531.

7 Schoch O, Schanz U, Keller M, et al. BAL findings in a patient with pulmonary alveolar proteinosis successfully treated with GMCSF Thorax 2002; 57: 277-280.

8 Burgio GR, Monafo V. Infectious mononucleosis fifty years after the discovery of the Paul-Bunnell test. Infection 1983; 11: 1-5.

\section{The fear of volcano: short-term health effects after Mount Etna's eruption in 2002}

\section{To the Editors:}

The dramatic volcanic eruption in Iceland has recently brought the possible health effects of volcanic ashes to the general attention of the media. Lessons learnt from other episodes may be of interest and could guide future public health action.

During Autumn 2002, a large eruption of Mount Etna (Sicily, Italy) occurred. It persisted for 3 months, showing peaks of intense volcanic activity characterised by explosions, jets hundreds of metres high and seismic activity. Local winds carried the ash columns for kilometres, causing discomfort to the population of Catania (nearly 370,000 inhabitants) and other inhabited areas near the volcano. Repeated episodes of rainfall occurred after the eruption, reducing the phenomenon of ash resuspension. The population was alerted by a general alarm caused by national and local mass media. National and local authorities enacted measures aimed at preventing health effects: schools were closed; motor vehicle circulation was prohibited; protective eye masks were distributed; the elderly and individuals affected by chronic diseases of the respiratory system were suggested to limit their outdoor exposure and to reduce physical activity.

An epidemiologic study [1] on acute health effects on the nearby population was conducted. During the study period (106 days of observation in 2002; 59 days of eruption and 47 days of noneruption) unusually high levels $\left(>200 \mu \mathrm{g} \cdot \mathrm{m}^{-3}\right)$ of particulate matter with a $50 \%$ cut-off aerodynamic diameter $<10 \mu \mathrm{m}$ (PM10) were recorded (fig. 1). A 284\% increase in PM10 and 385\% increase in daily levels of sulfur dioxide $\left(\mathrm{SO}_{2}\right)$ were observed with respect to the previous year. No changes in nitrogen dioxide $\left(\mathrm{NO}_{2}\right)$ and carbon monoxide $(\mathrm{CO})$ were detected. Earthquakes [2] started 1 day before the onset of the eruption, with at least two earthquakes per day (above magnitude 3) during the first week of eruption. As shown in figure 1, the intensity of seismic activity was highly correlated with PM10 levels.
Both mortality and hospital admissions data were collected for all residents of the city during eruption and noneruption days in 2002, using the corresponding days in 2001 as a control. Relative risks (RR) of health events during the eruption with respect to the control period were computed both for mortality and for hospital admissions, shifted 1 day forward in order to take into account the day of the week effect. No changes in allcause (RR 0.98, 95\% CI 0.86-1.12) and cardiovascular (RR 1.09, $95 \%$ CI 0.90-1.32) mortality were observed, whereas a significant decrease in mortality for respiratory diseases during the eruption period was detected (RR 0.46, 95\% CI 0.46-0.82). Hospital admissions for cardiovascular diseases significantly increased (RR 1.18, 95\% CI 1.08-1.29), particularly for ischaemic heart diseases (RR 1.31, 95\% CI 1.10-1.56) and for cerebrovascular diseases (RR 1.24, 95\% CI 1.05-1.47), especially among people aged $>65$ yrs. Also notable, although not statistically significant, were admissions for eye disorders, two times higher during the eruption period than in the previous year. However, a decrease in hospitalisations for trauma was observed (RR 0.9, 95\% CI 0.8-1.0).

To evaluate the time relationship between daily PM10 levels and daily hospital admissions for cardiovascular diseases, a time-series analysis with Poisson regression was conducted (considering day of the week and holidays as confounders) and evaluating different time lags for PM10 concentrations $\leqslant 14$ days. No statistically significant association appeared, although the effect increased with time lags suggesting a delayed effect on cardiovascular health.

Studies on health hazards related to volcanoes [3] have reported cardiovascular and respiratory problems possibly related to particulate matter and/or to emitted gases. In fact, volcanic ash is generally composed of particles of fragmented volcanic rock, most of which are within the coarse fraction range $(2.5-10 \mu \mathrm{m})$. Other minor or temporary health effects 